\title{
Sports physiotherapy - Actions to optimize the health of Para athletes
}

\author{
K Fagher $^{1}$ a, M Badenhorst ${ }^{2}$, P Van de Vliet $^{3}$ \\ 1 Department of Health Sciences, Rehabilitation Medicine Research Group, Lund University; The Medical Committee, The Swedish Paralympic \\ Committee, 2 Sports Performance Research Institute New Zealand (SPRINZ), Auckland University of Technology; Institute of Sport and Exercise \\ Medicine (ISEM), Division of Orthopaedic Surgery, Department of Surgical Sciences, Faculty of Medicine and Health Sciences, Stellenbosch University; \\ International Olympic Committee (IOC) Research Centres, ${ }^{3}$ Immun-oncological Centre Cologne \\ Keywords: Para sport, Physiotherapy, athlete health, athletic injuries, prevention \\ https://doi.org/10.26603/001c.29910
}

International Journal of Sports Physical Therapy

Vol. 16, Issue 6, 2021

\section{SPORTS PHYSIOTHERAPY - ACTIONS TO OPTIMIZE THE HEALTH OF PARA ATHLETES}

The interest and development of Para sport continues to increase around the globe. As Para athletes' performances and professionalism are steadily improving, there is also an increasing need and interest for athlete health and (para-)medical support. The term Para athlete refers to individuals with an impairment that participate in a sport that has classification rules as defined in the International Paralympic Committee (IPC) Athlete Classification Code (www.paralympic.org).

This calls for further scientific knowledge and professional competencies. We believe that physiotherapists are crucial partners in this regard. In this IFSPT perspective, we have summarized how sports physiotherapists can add value within various settings of Para sport.

\section{ASSESSMENT AND ATHLETE HEALTH MONITORING}

To increase the knowledge of Para athletes' health, it has been recommended to conduct athlete health monitoring on a regular basis. ${ }^{1}$ With experience in classifying health, functioning and disease, physiotherapists again can provide valuable knowledge into this area. The same applies to the assessment of injury. As indicated above, special attention is required for Para athletes given their uniqueness, both as individual athlete, and by nature of their injuries. Most recently, this was expanded upon in the area of concussion assessment, with a call for case-by-case decision-making. ${ }^{2}$

Athlete classification is a very specific application of assessment in which athletes are "classified" according to their ability to perform certain movements and tasks. ${ }^{3}$ Therefore, every athlete undergoes an extensive physical and sport-specific assessment. By nature of their qualification and skills, sports physiotherapists act as classifiers alongside physicians and sports experts across almost all Para sports. For more detail, the reader is referred to www.paralympic.org and Journal of Sports Sciences. ${ }^{4}$

\section{REHABILITATION OF SPORTS INJURIES IN PARA SPORT}

Para athletes report higher rates of sports injuries and illnesses compared to able-bodied athletes. ${ }^{5,6}$ For an athlete with an impairment, a sports injury or illness may not only affect sporting participation and performance, but also exacerbate the existing, underlying disability and compromise the athlete's ability to perform activities of daily life. ${ }^{4,7}$

Alongside injuries that often are similar to those of their able-bodied counterparts, Para athletes also report specific impairment- and equipment-related sport injuries. ${ }^{5,8}$ For example, poor proprioception can contribute to injuries in athletes with vision loss, whereas low bone mineral density and spasticity may increase the risk in athletes with neurological impairment. Therefore, it is important that the Para athlete is provided with strategies that can be applied both in sports and in daily life.

Thanks to a broad knowledge of musculoskeletal and neurological systems, sports injuries, the restoration of function and movement following an injury, and disability in general (impairments, activity limitations and participation restrictions), physiotherapists have an important role to play in supporting Para athlete health and welfare.

\section{PREVENTION OF SPORTS INJURIES IN PARA SPORT}

Physiotherapists are masters of the adage: "one size does not fit all.” For example, an athlete with transfemoral amputation or high spinal cord injury will not benefit from a regular knee control programme. Evidence-based physiotherapy focuses on tailored, patient-centred prevention

\footnotetext{
a Corresponding author:

Kristina Fagher, PhD, RPT, Department of Health Sciences, Rehabilitation Medicine Research Group, Lund University, PO Box 157 22100 Lund, Sweden. Tel: 004646222 1991, Fax: 004646222 1808, E-mail: kristina.fagher@med.lu.se
} 
programmes with an emphasis on behavioural science, health promotion and education, ${ }^{9}$ just what Para athletes need! In this sense, our profession has an important task to fulfill in the continuous development of holistic prevention strategies.

A very specific application of this knowledge is guiding athletes in their adaptive sports-specific equipment choice and its fitting (e.g. racing chair, sit-ski, running prosthesis). Thanks to the extensive knowledge of anatomy, ergonomics and assistive devices, physiotherapists will be able to assess the importance and impact of specific equipment and assistive devices as an additional component of medical care and prevention. ${ }^{4,10}$

\section{GETTING INVOLVED}

We advocate for sports physiotherapist engagement in Para sports:

- Sports physiotherapists are needed in the athlete support team. As a licensed health care practitioner with knowledge of disability and functioning, the musculoskeletal, nervous and cardiovascular system, and experience of working within multidisciplinary teams and biopsychological contexts, physiotherapists can advantageously take responsibility for the medical team during events and competitions.

- As academic professionals, sports physiotherapists play an important role to expand systematic data collection on injury and illness epidemiology in this population. Such data are needed to develop evidence-based treatment strategies, medical support, prevention programmes, and assessment methods.

- With classification moving into more evidence-based decision making, sports physiotherapists need to be involved in this process, as they are experts on human movement assessment.

- Sports physiotherapists must engage in increasing Para sport opportunities and share knowledge with fellow colleagues within the medical field.

Taken together, Sports Physiotherapists play an important role in understanding, promoting, assessing, maintaining, and restoring health in Para athletes around the globe. Ask yourself: are you doing your part?

Submitted: October 01, 2021 CST, Accepted: November 01, 2021 CST 


\section{REFERENCES}

1. Derman W, Badenhorst M, Blauwet C, et al. Para sport translation of the IOC consensus on recording and reporting of data for injury and illness in sport. Br J Sports Med. 2021;55(19):1068-1076. doi:10.1136/ bjsports-2020-103464

2. Weiler R, Blauwet C, Clarke D, et al. Concussion in para sport: the first position statement of the Concussion in Para Sport (CIPS) Group. Br J Sports Med. 2021. doi:10.1136/bjsports-2020-103696

3. Van de Vliet P. Let's talk facts - What healthcare providers really need to know about Paralympic athletes. Aspetar Sports Med J. 2016;5:74-79.

4. Mann DL, Tweedy SM, Jackson RC, Vanlandewijck YC. Classifying the evidence for evidence-based classification in Paralympic sport. Journal of Sports Sciences. 2021;39(sup1):1-6. doi:10.1080/02640414.20 $\underline{21.1955523}$

5. Fagher K, Dahlström Ö, Jacobsson J, Timpka T, Lexell J. Injuries and illnesses in Swedish Paralympic athletes-A 52-week prospective study of incidence and risk factors. Scand J Med Sci Sports.

2020;30(8):1457-1470. doi:10.1111/sms.13687
6. Derman W, Runciman P, Jordaan E, et al. High incidence of injuries at the Pyeongchang 2018 Paralympic Winter Games: a prospective cohort study of 6804 athlete days. Br J Sports Med. 2020;54(1):38-43. doi:10.1136/bjsports-2018-100170

7. Fagher K, Forsberg A, Jacobsson J, Timpka T, Dahlström Ö, Lexell J. Paralympic athletes' perceptions of their experiences of sports-related injuries, risk factors and preventive possibilities. Eur J Sport Sci. 2016;16(8):1240-1249. doi:10.1080/1746139 1.2016.1192689

8. Webborn N, Van de Vliet P. Paralympic medicine. Lancet. 2012;380(9836):65-71. doi:10.1016/s0140-673 $\underline{6(12) 60831-9}$

9. Fagher K. Sports-related injuries and illnesses in Paralympic athletes. Lund: Lund University: Faculty of Medicine, 2019. 118 s. (Lund University, Faculty of Medicine Doctoral Dissertation Series; 116).

10. Burkett B. Paralympic sports medicine--current evidence in winter sport: considerations in the development of equipment standards for paralympic athletes. Clin J Sport Med. 2012;22(1):46-50. doi:10.10 97/ISM.0b013e31824200a4 